\title{
Agôn
}

Revue des arts de la scène

HS 1 | 2011

Mettre en scène l'événement

\section{3 lycéens de Seine-Saint-Denis : Manhattan sur le plateau}

Entretien réalisé le 23 juillet 2011

Arnaud Meunier et Marion Boudier

(2) OpenEdition

Journals

Édition électronique

URL : http://journals.openedition.org/agon/1801

DOI : 10.4000/agon. 1801

ISSN : 1961-8581

Éditeur

Association Agôn

Référence électronique

Arnaud Meunier et Marion Boudier, " 43 lycéens de Seine-Saint-Denis : Manhattan sur le plateau », Agôn [En ligne], HS 1 | 2011, mis en ligne le 02 septembre 2011, consulté le 15 septembre 2020. URL http://journals.openedition.org/agon/1801 


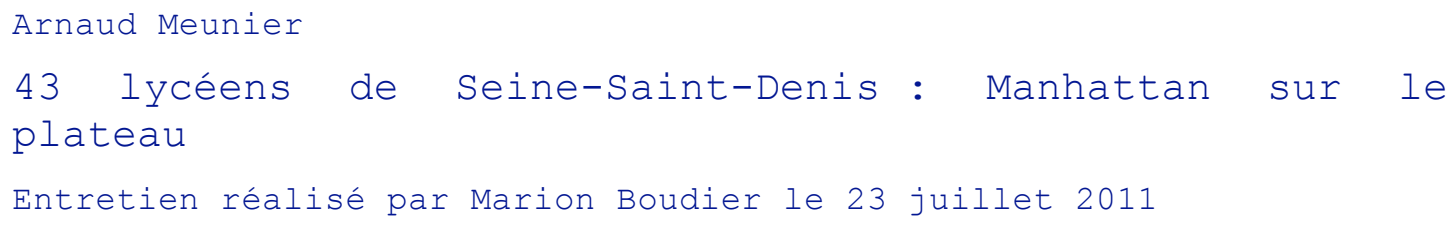

Diplômé de Sciences Politiques, Arnaud Meunier (1973) a suivi une formation de comédien avant de fonder la Compagnie de la Mauvaise Graine en 1997 (www.ciemauvaisegraine.com). Depuis janvier 2011, il dirige La Comédie de Saint-Étienne, Centre dramatique national. Il a travaillé pour des opéras en tant que metteur en scène ou dramaturge, et dans de nombreux pays. Formateur, il défend la nécessité d'une éducation artistique ouverte au plus grand nombre. Dans cet entretien, réalisé quelques semaines avant la création en partenariat avec le Théâtre de la Ville à Paris, il revient sur la genèse et le déroulement du projet «D'un 11 septembre à l'autre ", sur son engagement civique auprès des amateurs et sur son travail de metteur en scène avec ses comédiens et les lycéens de Seine-Saint-Denis pour monter 11 septembre 2001 de Michel Vinaver.

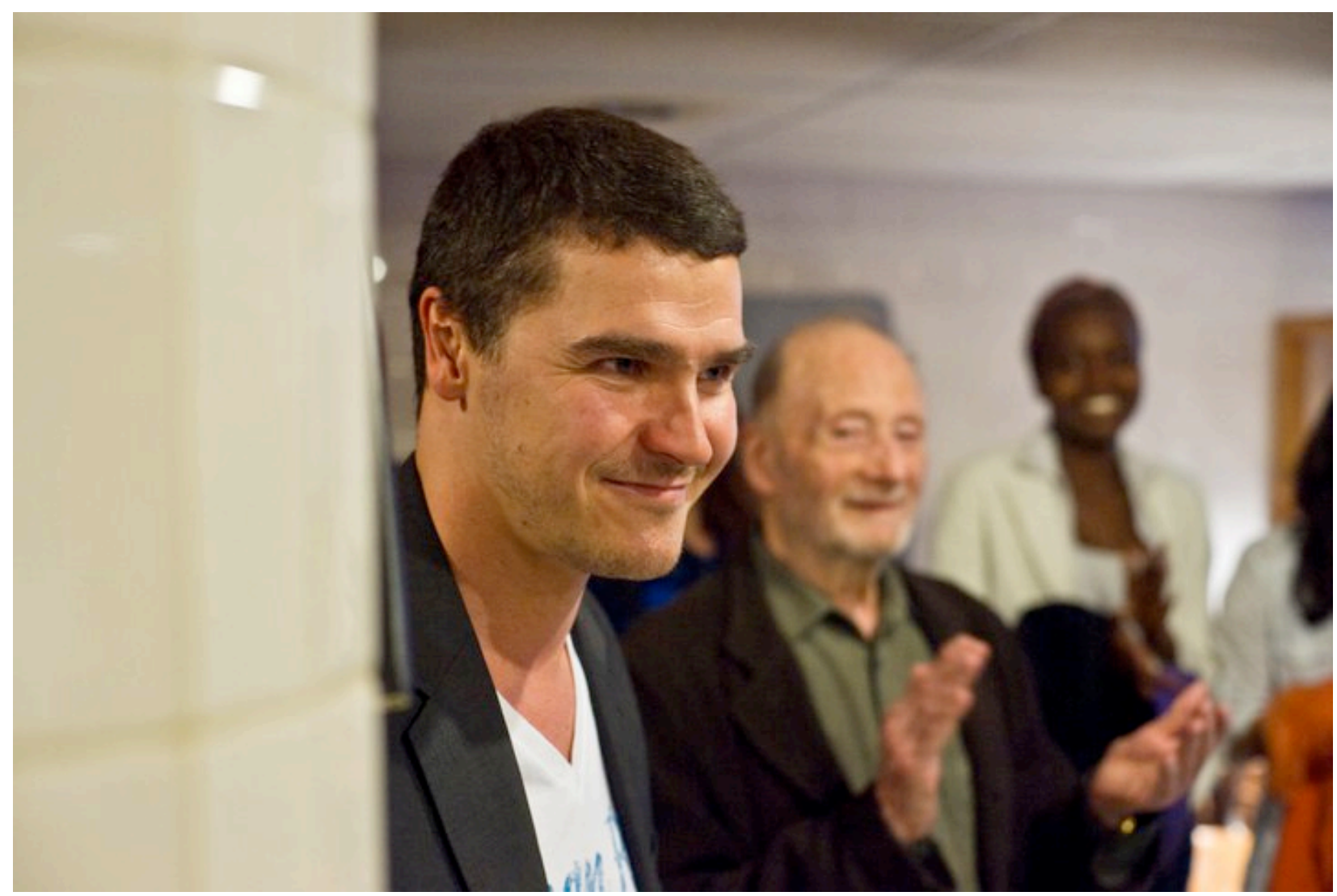

Arnaud Meunier félicitant les lycéens après la présentation au public du travail le 15 avril 2011 au Forum de Blanc-Mesnil Michel Vinaver en arrière-plan. (C) Pierre-Etienne Vilbert 


\section{Genèse du projet}

Marion Boudier - Le projet "D'un 11 septembre à l'autre » a été initié par la compagnie de production cinématographique Veilleur de nuit qui a sollicité ta compagnie de théatre, la Mauvaise Graine. Ta proposition de travailler avec des lycéens en Seine-Saint-Denis a amené un nouveau partenaire au projet, l'association Citoyenneté Jeunesse, qui vous a accompagné dans le choix des lycées puis des classes dans les villes de Noisy, Aulnay et Bondy. Cette genèse estelle exacte?

Arnaud Meunier - Oui. Quand Veilleur de nuit m'a sollicité, j'étais en train de travailler sur Le Problème de François Bégaudeau avec Emmanuelle Devos et Jacques Bonnaffé notamment, donc j'imagine que Veilleur de nuit ne s'attendait pas à ce que je propose un casting de lycéens ! Ce qui est beau, c'est que ce qui aurait pu faire reculer un producteur privé a reçu de leur part un très grand intérêt.

M. B. - Ce projet s'inscrit à la fois dans le prolongement de ton travail sur M. Vinaver - tu as monté La Demande d'emploi, King et l'adaptation de Par-dessus bord par O. Hirata - et dans celui de ton travail avec des amateurs, puisque tu avais déjà été en résidence en Seine-SaintDenis, au Forum du Blanc-Mesnil de 2000 à 2003, où tu avais mené avec ta compagnie de nombreux ateliers pour "propager un désir de théâtre ».

A. M. - Oui, mon travail de compagnie s'est toujours appuyé sur la création et sur ce que j'appelle des " actions de sensibilisation ». " Action culturelle » est un terme trop générique. Les membres de ma compagnie ne sont pas issus du milieu théâtral, ce ne sont pas des « gens de la balle »; nous sommes arrivés au théâtre grâce à des portes d'entrée que nous souhaitons continuer à ouvrir pour d'autres.

\section{Une pièce-matériau}

M. B. - On peut faire des liens entre les pièces de M. Vinaver que tu as précédemment montées et 11 septembre 2001 : l'évocation de l'Amérique (du krach boursier de King au crash des avions), l'inclusion d'éléments documentaires dans une poétique de l'ordinaire, qui témoigne de l'histoire sans vouloir être un discours de témoignage... Quelle place particulière 11 septembre 2001 occupe-t-elle dans ton rapport à l'ouvre de M. Vinaver?

A. M. - 11 septembre 2001 est effectivement une pièce à part par rapport aux trois autres que j'ai montées: c'est une pièce-matériau. Elle devait servir de livret pour un opéra [de G. Aperghis], qui ne s'est finalement pas fait. La pièce garde donc un aspect matériau dans la mesure où sa dramaturgie est plus éclatée que celle des autres pièces, dans lesquelles la dramaturgie me semble plus immédiate. Cela m'a donné une liberté. Dans le spectacle, par exemple, on répète deux ou trois fois certains témoignages : je me suis autorisé cette répétition, ce que je ne me serais pas autorisé sur les autres pièces. Il me semblait également important que les passages du chœur, qui sont en anglais, soient sous-titrés, pour les lycéennes qui les jouent et pour leurs familles. M. Vinaver dit que c'est de la matière sonore musicale, que ce n'est pas grave si les gens ne comprennent pas, mais je trouve que cela créé une injustice entre les spectateurs anglophones et ceux qui n'ont aucune notion de ce que jouent le chœur. Les portes d'entrée pour la mise en scène sont différentes de celles des autres pièces, c'est pour cela que je parle de pièce-matériau.

11 septembre 2001 est également une pièce très brève. Elle n'a rien à voir avec La Demande d'emploi qui est très architecturée, avec une progression en hélice, ou avec le parcours de King. Le seul point commun, c'est que c'est toujours un défi de mettre en scène une pièce de $M$. Vinaver! La dramaturgie n'est pas académique, elle pose mille et une questions au metteur en 
scène et aux acteurs : c'est ce qui pour moi est toujours un moteur d'enthousiasme. Ce qui est intéressant avec les lycéens, c'est qu'ils ne posent pas les questions que posent d'habitude les comédiens sur les pièces de M. Vinaver. Ils n'ont pas été gênés par la forme de la pièce, l'absence de situation, et en même temps, le fait qu'il fallait prendre en charge chaque témoignage, que la parole devait réellement être entendue, crédible, etc., était très clair pour eux. Je le dis souvent: quand on s'adresse à des publics qui n'ont pas d'a priori culturels, la liberté artistique est plus grande.

\section{1 septembre 2001 et le 11 septembre 2001}

M. B. - La pièce 11 septembre 2001 a-t-elle modifié ton rapport personnel à l'événement 11 septembre 2001 ?

A. M. - Fondamentalement, la pièce n'a pas changé mon rapport à l'événement. Au départ, on avait un fort préjugé autour de l'idée que les jeunes seraient très sensibles aux théories du complot. Pas plus qu'ailleurs en fait. Le fantasme selon lequel les jeunes gens des banlieues, de confession musulmane, sont plus perméables à ce genre de discours est erroné.

M. B. - Dans la note d'intention du projet, tu évoques le 11 septembre 2001 comme une " matrice originelle d'où découlent encore aujourd'hui des actes et des positions qui construisent notre quotidien ». Mais une des enseignantes qui a accompagné les lycéens sur le projet mentionne "la minceur de leurs souvenirs"; ils avaient en effet à peu près 7 ans en 2001. Ils n'avaient donc peut-être pas tout à fait conscience de l'impact politique de cet événement. Cela a-t-il été une surprise pour toi ?

A. M. - Oui, une surprise et, en même temps, une surprise rassurante, à l'inverse du fantasme : ces lycéens sont comme le reste de la population. Ils avaient tout de même une conscience assez forte du lien entre le 11 septembre et la guerre en Irak, entre cette guerre et les intérêts économiques, notamment pétroliers, et une conscience de l'équation qui peut en découler arabes $=$ musulmans $=$ terroristes. Ils avaient une conscience globale de cela, mais sur un fond un peu imprécis, que nous avons découvert en cours de travail. Ce qui est clair pour moi, c'est qu'avec le week-end du 11 septembre 2011 on clôtura la première décennie du siècle. Le 11 septembre nous fait entrer de plain-pied dans le $\mathrm{XXI}^{\mathrm{e}}$ siècle ; ce premier anniversaire va nous faire tourner la page de la première décade. Cet événement est structurant pour la décennie que l'on vient de vivre.

M. B. - "Tourner la page ": tu es effectivement tourné vers l'avenir avec ce projet.

A. M. - Exactement, le principe artistique du spectacle est de se dire que là où tout le monde va être dans un mouvement commémoratif, nous serons dans un mouvement inverse, tournés vers l'avenir.

\section{L'éveil d'une conscience politique}

M. B. - As-tu l'impression que les répétitions de ce spectacle, en déclenchant une prise de connaissance et une forme d'appropriation de l'événement par les lycéens, a entraîné l'éveil d'une conscience politique?

A. M. - Je pense que oui. C'est un aspect positif. Comme dans toute la jeunesse, le niveau de conscience politique, d'engagement ou de militance est extrêmement variable d'un lycée à l'autre. Certains d'entre eux étaient déjà des gens plutôt militants, avec un vrai point de vue sur un certain nombre de choses. Ce qui est clair, c'est que le travail pédagogique mené par Citoyenneté Jeunesse en parallèle de notre travail artistique a contribué à construire des outils de réflexion. Nous ne voulions absolument pas avoir une démarche messianique. Nous n'allions pas cultiver des jeunes gens acculturés ou incultes, mais leur offrir la possibilité de découvrir un 
champ artistique dans lequel ils pouvaient trouver du plaisir, un plaisir complémentaire d'autres pratiques qu'ils ont par ailleurs. Je défends fortement l'idée qu'on peut aimer les jeux vidéo, être un fanatique de séries américaines et prendre plaisir à aller voir du théâtre. Citoyenneté Jeunesse a fourni des outils conceptuels; la séance la plus frappante a été pour moi celle de l'ethnologue Mourad Hakmi qui a construit avec eux des concepts - qu'est-ce qu'être arabe, qu'être musulman, est-ce la même chose, qu'est-ce que l'Islam ?, etc. - et a déconstruit les théories du complot. Cela a été mené sur un mode socratique ; on leur a donné des éléments de réflexion pour qu'ils soient libres ensuite de les agencer.

M. B. - Leur réflexion a dû également évoluer en lien avec l'actualité. Je pense à la mort de Ben Laden notamment.

A. M. - Oui, bien sûr. Quand la mort de Ben Laden est arrivée, leur réaction a été peu ou prou celle de toute l'équipe encadrante, c'est-à-dire une forme de soulagement d'une part, et une forme de stupéfaction d'autre part, sur le côté expéditif de l'affaire et l'absence de justice, de procès. Ce que j'avais sous-estimé également, c'est le rapport affectif aux témoignages de la pièce de M. Vinaver, le fait de se projeter (" et si ça avait été moi ») est très puissant dans la construction de leur réflexion. L'alliance du travail pédagogique et du travail artistique est essentielle. Du point de vue des enseignants aussi, l'acquisition de certains outils qu'ils n'utilisent pas en classe a été importante. Les professeurs d'économie ou d'histoire ont particulièrement apprécié la séance avec l'ethnologue, qui leur a permis de travailler d'une autre façon que celle qu'ils s'autorisent d'ordinaire. Les enseignants ont peur d'aborder ces thématiques en classe, mais l'étude simple du fait historique du 11 septembre 2001 ne permet pas d'aborder les concepts dont il a été question.

M. B. - Il me semble que cela est lié aussi à la dramaturgie de M. Vinaver, qui est à l'opposé d'un théâtre à thèse et offre la possibilité d'aborder les faits avec une grande liberté de jugement.

A. M. - Oui, tout à fait. Et avec de l'humour également. Par exemple, j'ai distribué le jeune Fouad dans les rôles de Mohammed Atta et de Ben Laden, car il a une qualité d'investissement et d'émotion très forte ; il est croyant et cette croyance nourrit sa parole. À un moment, je me suis quand même demandé si ce n'était pas politiquement dangereux, si je n'étais pas en train de lui faire jouer « l'arabe de service ». On en a parlé, cela ne lui posait pas de problème ; il avait tout à fait conscience que je n'étais pas en train de l'enfermer dans un cliché. Il y a une telle confiance entre les membres de l'équipe que j'ai fait la distribution en fonction des points forts de chacun sans me poser d'autres problèmes.

\section{L'activité artistique, une exigence}

M. B. - En tant qu'artiste, directeur d'une compagnie qui travaille souvent avec des amateurs et des jeunes de banlieue, sans parler de "messianisme", tu es donc très conscient de ta responsabilité ou de ta mission civique.

A. M. - Je pense que notre mission, c'est de faire connaître une pratique à des gens qui ne la connaissent pas et ne savent donc pas encore qu'ils pourraient l'aimer. Pas dans un geste prosélyte, bien sûr.

M. B. - N'es-tu pas aussi attaché à changer l'image que certaines personnes peuvent avoir des jeunes de banlieue?

A. M. - Clairement.

M. B. - Ce projet va sans doute être extrêmement médiatisé.

A. M. - On l'espère. Ce territoire m'a tellement porté artistiquement. On a parlé du BlancMesnil, mais on pourrait évoquer le Théâtre Gérard Philipe de Saint-Denis, La Commune d'Aubervilliers. C'est aussi un département qui m'a soutenu, subventionneur régulier et fidèle. 
Pour moi, c'est donc toujours une surprise de me rendre compte du décalage entre l'image médiatique du département et la réalité du terrain. Je ne dis pas que c'est un département simple. De plus j'y travaille, je n'y vis pas. Mais je suis toujours frappé par son énergie. C'est le département le plus jeune de France, celui où il y a le plus de nationalités représentées, les enseignants y sont d'une grande qualité. L'immense majorité des gens que j'y ai rencontrés sont inventifs, militants, à la cherche de meilleures façons d'aider les jeunes, refusant les déterminismes. Je trouve par exemple le partenariat avec Sciences Po passionnant. Je pense qu'une jeune fille de notre équipe tentera cette école. Sinon, tous les élèves impliqués passent en terminale, sauf un qui va se réorienter. La classe de $1^{\text {ère }}$ STG, qui nous paraissait au début du projet comme celle qui en était peut-être la plus éloignée, participe finalement avec plus de la moitié de ses élèves à la création du spectacle. Les enseignants ont constaté une amélioration des résultats scolaires, un meilleur « raccrochage » pour ceux qui avait un peu délaissé les études. Cela continue de me convaincre que l'activité artistique doit intervenir vite et tôt dans le parcours. Le projet a été bénéfique pour l'ensemble des élèves dans leur rapport à la concentration, aux enseignants, au collectif, leur capacité à travailler ensemble, l'estime qu'ils ont d'eux-mêmes... Les élèves de STG qui ne participent pas à la mise en scène restent attachés au projet; ils ont écrit des textes, ils ont participé à la préparation de la présentation au BlancMesnil en avril. Il y a un phénomène de rayonnement qui dépasse les lycéens qui sont sur le plateau. Quand je parle d'absence de messianisme, c'est pour dire que si on a des a priori condescendants sur la sous-culture de ces jeunes, victimes de la consommation de masse, dont ils n'auraient pas conscience, on est dans la caricature. Certains sont très conscients. Citoyenneté Jeunesse est, par exemple, en train de fabriquer un journal sur le modèle du New York Times, qui sera distribué aux spectateurs, dans lequel Abdi témoigne avec beaucoup de recul des contrôles au faciès dont il est victime. C'est plus difficile pour d'autres qui sont dans des situations personnelles plus dures. Et bien sûr, le fait qu'ils soient des fans de slam ou écoutent du hip hop n'est absolument pas contradictoire avec le fait de jouer du Vinaver.

M. B. - Est-ce qu'ils ont influencé les choix de musique pour le spectacle?

A. M. - Un peu, mais ils n'ont pas construit la musique. Maintenant, c'est le spectacle qui est à construire. Il nous reste trois semaines de répétitions intensives. Ils ont déjà travaillé cent heures en atelier et deux semaines en répétition. Mis bout à bout, c'est un temps conséquent : cent heures et cinq semaines. Les enseignants sont inquiets, car ce ne sont que des adolescents, qui ne sont pas habitués aux rythmes de travail. Je pense le contraire : ils sont en demande. Ce qui a tiré le projet vers le haut, c'est l'exigence qu'on a pour eux. Cette exigence-là leur raconte qu'ils en sont capables, et qu'ils le méritent. Cette exigence les motive. Une des questions qui est revenue le plus souvent, c'est pourquoi nous, pourquoi avons-nous été choisis ?

M. B. - Qu'est ce que tu leur réponds?

A. M. - Qu'on a choisi des lycées, avec des équipes pédagogiques motivées, et que ce sont leurs enseignants qui les ont choisis, eux. La participation des enseignants est très importante. D'ailleurs, c'est touchant de voir qu'ils se sont tous organisés pour pouvoir venir à Saint-Étienne pendant les répétitions du mois d'août.

\section{Déroulement des ateliers}

M. B. - Pourrais-tu m'expliquer exactement quel a été le fonctionnement des ateliers. Ils ont été menés par cinq comédiens de la Mauvaise Graine, Philippe Durand, Elsa Imbert, Nathalie Matter, Stéphane Piveteau et Thierry Vu Huu. Y assistais-tu? Où la mise en scène a-t-elle commencé à s'inventer? 
A. M. - Une fois que les lycées et les équipes enseignantes ont été choisis - j'insiste sur les équipes, car nous avions commencé à travailler avec un quatrième lycée avec lequel nous avons dû abandonner faute d'équipe pédagogique soudée -, puis une fois que les classes ont été choisies, on a rencontré les élèves pour leur présenter le projet. On a fait un mini-stage de trois jours, obligatoire, pour chaque classe, organisé dans un lieu partenaire. On les a sensibilisés au théâtre et au projet. Ensuite, les comédiens se sont répartis sur les trois établissements, en subdivisant les classes nombreuses, ce qui a créé cinq groupes. Un comédien s'occupait donc d'un groupe. Je n'assistais pas à ces séances d'ateliers, mais nous nous étions mis d'accord et les comédiens envoyaient des mails collectifs chaque semaine pour faire un bilan. Nous pouvions réagir en continu les uns par rapport aux autres.

M. B. - C'était donc du travail de plateau.

A. M. - Un travail d'initiation : c'est quoi être sur un plateau, quel est le rapport à la voix, à l'autre, comment vaincre la peur du ridicule, se laisser regarder, oser. Sans aucun texte. Nous voulions travailler sur la conscience du corps, en laissant la parole de côté. En novembre, a eu lieu un premier week-end d'atelier collectif : une centaine de lycéens qui devaient apprendre à se rencontrer. Nous avons travaillé sur l'apprentissage de l'altérité et la prise de risque, le lâcher prise. Toujours sans texte. Après ce week-end, nous avons commencé à aborder le texte, avec une phrase, puis deux, etc., qui n'étaient pas tirées du texte de M. Vinaver. Tout le début de l'année a été consacré aux bases fondamentales du théâtre. À partir de janvier, on a commencé à travailler sur 11 septembre 2001, et les comédiens ont élaboré des distributions lors de leurs ateliers hebdomadaires, en attribuant des bouts de textes à certains élèves, selon les envies. Lors du premier week-end de janvier, Rachid Ouramdane leur a fait travailler le corps de manière plus poussée, et j'ai travaillé à partir des choses qu'ils avaient apprises. Lors du week-end de février, je les ai écoutés dans la totalité de ce qu'ils avaient appris. Ils étaient fiers de montrer ce qu'ils savaient, tout en ayant très peur! C'est à ce moment-là que certains ont compris qu'ils ne voulaient pas aller sur le plateau, et qu'on a commencé à voir qui irait jusqu'au bout. Certains étaient très motivés, avec l'envie de jouer des personnages précis. Une émulation s'est créée.

M. B. - Y avait-il des propositions de mise en scène à cette étape du travail ?

A. M. - Non, ils étaient juste concentrés sur la connaissance du texte et sur leur capacité à être crus. Ils avaient travaillé en ateliers sur le rapport sensible à la parole, comment interpréter un personnage plus âgé, qu'est-ce que ça veut dire être cru, à quelle vitesse on parle, etc. La semaine des vacances d'avril a été déterminante. Ils étaient en vacances, ce sont donc les plus motivés qui sont venus. Le groupe a pris forme.

\section{Un « tout-monde »}

M. B. - J'aimerais revenir sur cette question de l'oralité, sur laquelle, d'ailleurs, tu insistes dans ta note d'intention ("ce qui fut l'oralité du 11 septembre $2001 »)$. Il y a, en effet, une variété de régimes de parole dans le texte de M. Vinaver, texte bilingue qui est un montage de paroles rapportées, de voix aux tonalités différentes (propos quotidiens, discours, etc.) plus ou moins médiatisées (téléphone, radio, extrait de presse, etc.) et qui ne partagent pas le même espace. Comment as-tu travaillé sur cette oralité avec les lycéens? Ces jeunes amateurs avaientils un rapport particulier à la langue - un rapport moins sacré peut-être-ou au rythme?

A. M. - Je pense sincèrement qu'ils sont les meilleurs interprètes possibles de cette pièce, qui prend une dimension et une profondeur très puissantes à travers la vitalité de leurs corps et dans leurs voix. À eux tous, ils représentent un «tout-monde », selon la formule d'Édouard Glissant, comme les tours jumelles représentaient un monde. L'immense diversité de leurs origines, même s'ils sont tous Français, couvre presque la planète entière : les Caraïbes, les Antilles, l'Afrique, 
l'Amérique du sud, le Maghreb, le Pakistan, la Turquie, la Slovaquie, la Croatie, l'Espagne, le Portugal, la Belgique et des Gaulois de souche aussi. Le rapport à la parole a été essentiel. Ils ont senti qu'ils avaient une responsabilité de faire revivre ces témoignages. Ils ont également fait preuve de beaucoup d'intelligence sur la similarité des discours à la fin, sur la portée politique qu'il y a à faire entendre cette similarité. Par rapport à l'anglais, ils étaient un peu réticents au début, car ils avaient peur de ne pas être compris ; la décision de surtitrer les passages du chœur a réglé ce problème. Les professeurs d'anglais ont eu un très bel engagement également. Ces jeunes ont de toute façon un rapport au monde très ouvert, lié à l'éclatement des familles qui ont immigré. Ils ont un rapport à la globalisation.

M. B. - Le texte est en partie construit sur une tension entre l'individu et le collectif, des voix singulières et un chœur. M. Vinaver fait entendre des voix anonymes, singulières, parce qu'ils voulaient résister au discours collectif qui allait, en quelque sorte, figer l'événement dans des stéréotypes. Finalement, de mon point de vue de spectatrice [de l'étape de travail d'avril], ces voix singulières, parce qu'elles touchent à quelque chose d'universel, retissent une communauté, inventent une nouvelle collectivité.

A. M. - Oui, c'est juste.

M. B. - Mais pourquoi ce nombre, quarante? Avais-tu dès le départ des images de foule sur scène en tête?

A. M. - Dès le départ, je voulais une cinquantaine de jeunes sur le plateau. C'est pour cela que les enseignants ont proposé de commencer avec cent vingt lycéens - en fait, on a commencé à quatre-vingt-cinq, puisqu'on a perdu un lycée au départ. Comme la pièce est chorale, je voulais pouvoir travailler sur la masse. Il me fallait pouvoir travailler sur la masse et sur la diversité, la diversité des corps, des origines, pour que ça fasse Manhattan sur le plateau. Quand on lit la pièce, ce qui frappe ce sont les noms propres des personnages qui reflètent toutes les origines et les signes de solidarité. Le premier réflexe n'a pas été la panique mais l'entraide; cette thématique a beaucoup touché les lycéens. J'avais deux principes de travail fondamentaux : la compagnie éphémère et la bienveillance. La bienveillance est un principe très actif, en osmose avec la pièce de Michel.

M. B. - C'est très fort quand un jeune se détache du groupe pour prendre la parole. Je suppose que la bienveillance du groupe est essentielle dans ces moments.

A. M. - C'est très important. On a décidé par exemple de donner le témoignage de John Paul DeVito, qui est un texte important et long, à un seul jeune, qui s'appelle Soumail et qui est d'origine mauritanienne. C'est une responsabilité. La pièce est une partition, qui permet des solos et un travail choral, avec des énergies très différentes : ça n'a rien à voir d'être cinquante sur le plateau ou deux ou trois. Lorsque tout à coup le plateau se vide, la capacité d'écoute et l'énergie sont très différentes.

M. B. - Comment avez-vous travaillé ces passages, c'est-à-dire la formation, la dissolution et la recomposition permanente du groupe? Soudain, je pense à ton travail antérieur sur les textes d'O. Hirata dans lesquels le groupe est une sorte d'organisme vivant en permanente évolution, avec des simples traversées du plateau, des moments de vide et de foule.

A. M. - Ils ont adoré le travail de groupe. C'était important d'avoir des moments où tous travaillaient ensemble. Bien sûr, le lien est très net avec mes spectacles précédents, par rapport au texte partition, à la gestion de la foule, à la manière dont la banalité fait sens, par rapport aussi à l'absence de didactisme qui ouvre le questionnement et le politique... Il y a effectivement un fourmillement dans la pièce de $\mathrm{M}$. Vinaver, et la référence au chœur antique bien sûr. Au final, la moitié des lycéens qui sont sur le plateau sont de confession musulmane. C'est très intéressant que le week-end du 11 septembre, pour les dix ans, ces témoignages soient portés par de jeunes musulmans: le symbole de fraternité et d'ambition qu'envoie le spectacle est pour moi très puissant. 


\section{L'espace et la métamorphose des corps}

M. B. - Quel type d'espace avez-vous finalement élaboré avec le scénographe?

A. M. - Contre toute attente, il y a un espace scénographié alors qu'au début je voulais une foule pour que les corps créent le décor. Au fur et à mesure des séances de travail, j'étais tellement heureux et fier de ce qui se passait, et par ailleurs très agacé par les réactions du milieu et des financeurs frileux par rapport à l'accès d'amateurs à des scènes professionnelles, que je me suis dit que pour que le spectacle soit vécu comme une création à part entière, il fallait qu'il soit payant et non gratuit d'une part, et, d'autre part, que la forme spectaculaire soit aussi aboutie que dans n'importe quel autre de mes spectacles. Donc, en cours de route, j'ai décidé de faire faire un décor. Il y aura également des costumes. Comme toujours dans les textes de M. Vinaver, étant donné que les pièces sont éclatées, on a besoin de niveaux, de différentes hauteurs, de manière à pouvoir localiser la parole. Pour savoir qui parle, il faut que le costume, la situation, le racontent.

M. B. - Je dirais plutôt d'où ça parle, parce qu'on sait qui parle, étant donné que les noms des personnages doivent être dits - c'est ce que préconise la note liminaire de la pièce.

A. M. - Qui parle et d'où ça parle, effectivement. C'est amusant, car pour savoir qui parle, il y a plusieurs possibilités à chaque fois. On a élaboré un décor qui est un espace en reconstruction, qui peut être l'évocation de la reconstruction de Ground Zero ou celle de la destruction, de la même manière que cela peut aussi évoquer les cours d'immeubles et de cités de la Seine-SaintDenis. Il s'agit donc d'une sorte d'échafaudage, avec un traitement de couleur qui évite le naturalisme. Cela peut aussi évoquer la comédie musicale, West Side Story... Le chœur est traité à la Broadway de façon évidente. Je suis donc en train de construire un véritable spectacle, avec décor, lumière, musique, etc. Je ne pouvais pas faire moins par rapport aux lycéens. Cet engagement est aussi une des garanties de celui des lycéens; ils ne peuvent pas faire les choses à moitié.

M. B. - Pourrais-tu me parler du travail chorégraphique et gestuel mené avec Rachid Ouramdane et Jean-Baptiste André? Les corps ne feront donc finalement pas décor, mais le rapport des corps à la figuration et à l'image est une question importante soulevée par ce spectacle.

A. M. - Les sociétés occidentales oublient le corps ; il y a peu de gens qui ont conscience de la réalité de leur corps. On vit dans des sociétés où l'on prend peu soin de nos corps: pas de massage, pas de cérémonie de préparation au mariage comme on peut le faire au Maghreb, pas de sensualité... Cette prise de conscience est importante. Le théâtre n'est pas que dans le texte, il faut incarner, avoir un rapport au corps. J'ai voulu faire équipe avec Rachid Ouramdane en raison de son travail sur l'identité ; le travail chorégraphique de Rachid Ouramdane me semblait croiser sur le plateau le travail que je mène d'un point de vue théâtral. Au départ, il a travaillé sur des évidences en lien avec l'événement: course, chute, déséquilibre, mouvement d'entraide, de solidarité, pouvoir se toucher. Rachid Ouramdane ayant dû réduire son investissement dans le projet pour des raisons de santé, Jean-Baptiste André, qui vient du cirque, a poursuivi, en accentuant le travail sur le déséquilibre. L'idée n'est pas de rendre les corps dansants mais expressifs, de façon à pouvoir créer des architectures. J'ai orienté aussi le travail vers la question de la transformation et de la métamorphose : qu'est-ce qui se passe quand ils sont en sweat à capuche, puis apparaissent en costume avec cravate? Le regard du spectateur va évoluer dans le spectacle, c'est l'occasion pour moi de travailler sur le préjugé. Je travaille sur le trouble du spectateur. Bien sûr, pas de métamorphose pour les vieillir ; ils amènent une distanciation très puissante. L'âge et l'accent malien de la jeune fille qui joue Katherine Ilachinski donnent des frissons ; la théâtralité est sensible, cela a été immédiat. 


\section{Le réel, jusqu'aux frissons}

M. B. - On a tous vu des images des tours, mais pas de ce qui s'est passé dans les avions. Or c'est ce que fait entendre et donc voir la pièce.

A. M. - On a besoin de sensations, de figures. Il faut que l'hôtesse de l'air ressemble à une hôtesse de l'air. Il n'y aura pas d'images de l'événement, car je pense que tout le monde a ces images en tête : la puissance d'évocation du théâtre est plus forte que celle de l'image. Les seules images qu'on verra à la fin sont des photos des lycéens enfants. Ce qui m'intéressait, c'était aussi ce rapport 7-17 ans ; l'événement a marqué leur vie de manière indubitable. Ce spectacle permet de travailler sur le regard et sur le préjugé, mais aussi sur les conséquences, sur leur vie de manière profonde.

M. B. - Dans tes spectacles précédents, tu travaillais sur une forme de réalisme décalé, avec des effets de reconstitution et d'étrangeté. J'ai l'impression qu'avec ce projet, il ne s'agit pas de travailler avec le réalisme mais de travailler avec le réel, le réel des éclats de paroles rapportés, le réel de la vitalité et de la diversité des lycéens, le réel du plateau... Comment préserver ce réel ? Comment être vrai dès lors qu'on est sur une scène qui, quoi qu'on veuille, déréalise?

A. M. - Oui, mais cette question ne s'est quasiment plus posée étant donné leurs corps et leurs voix, et étant donné la prise de risque. Dans les scènes de métamorphose, quand ils changent de costumes, ils y vont à fond, ils se déshabillent vraiment. Le réel apparaît par leurs vibrations à eux. Leur engagement crée le réel. On n'a jamais entendu les paroles de cette pièce comme cela. La difficulté en conséquence, c'est que les cinq acteurs sonnent faux, terriblement faux. Il y a une telle vérité dans l'engagement des jeunes gens, que j'ai décidé que les acteurs joueraient le journaliste, qui est un commentaire sur ce qui se passe - la parole est identifiée selon cette valeur, donc ça fonctionne. L'artifice de l'acteur est sensible alors que chez les lycéens, il y a une immédiateté qui ne donne pas l'impression qu'ils fabriquent, et cela donne une puissance inouïe au texte pour moi. On espère que la grande salle du Théâtre de la Ville n'étouffera pas cette puissance.

M. B. - Devant ces acteurs qui n'incarnent pas psychologiquement mais ne sont pas non plus dans la distance de porte-paroles, j'ai été troublée en effet. J'ai ressenti une forme de pureté, mais j'hésite à employer ce mot... Ils ne fabriquent pas, ils sont eux-mêmes dans cette parole.

A. M. - Plutôt que pureté, je dirais qu'ils sont à l'état brut. De la matière brute, palpitante de vivant qui donc nous émeut parce qu'elle nous touche sans intermédiaire, sans artifices.

M. B. - Vous n'avez pas travaillé à les faire devenir acteurs.

A. M. - Exactement. Par contre, on les a fait travailler sur le fait qu'ils devaient être concernés par la parole qu'ils donnaient. On a des frissons parce qu'on y croit. Quand un jeune garçon raconte que les passagers vont attaquer les terroristes dans le quatrième avion et demande à la standardiste de dire à sa femme et à ses deux enfants qu'il les aime, on y croit, même s'il a dixsept ans. C'est la magie du théâtre. La crédibilité ne vient pas du réalisme ou du naturalisme, mais de l'implication émotionnelle de celui qui parle.

M. B. - Mon expérience de spectatrice est effectivement celle d'un grand frisson, l'impression d'une justesse qui n'est pas liée à un sentiment d'empathie que l'on peut parfois ressentir face au réalisme ou à l'authenticité de certains témoignages, mais à un saisissement, une sorte de fusion, mêlée à la distance que crée leur âge...

A. M. - Je pense que ce spectacle parle tout autant du 11 septembre 2001 que du 11 septembre 2011. Ça parle d'aujourd'hui, de ce qu'ils sont, tout en nous renvoyant à l'événement. L'écho est d'autant plus fort que ça nous rappelle l'événement et nous interroge très précisément sur aujourd'hui. 


\section{L'avenir}

M. B. - "Et maintenant»?

A. M. - C'est la grande question. Je suis parti de cette fin du texte pour construire le spectacle. C'est ce « et maintenant et maintenant et maintenant» qui a construit tout mon travail.

M. B. - Ton approche de la pièce est résolument tournée vers l'avenir - "C'est un support pour interroger aujourd'hui et la suite ", peut-on lire en effet dans ta note d'intention. Mais les représentations vont avoir lieu à un moment commémoratif, moment d'un travail de mémoire, tourné vers le passé. Je comprends bien comment ton souci de l'aujourd'hui et du futur a pu guider tout au long de l'année un parcours culturel et pédagogique, aider à construire un processus de travail et une dramaturgie : où cela se voit-il dans le spectacle? Tu as parlé d'une scénographie qui pouvait évoquer les cités. Y a-t-il des moments particuliers du spectacle où tu penses montrer le " maintenant » et l'avenir?

A. M. - Oui, cela est lié à ce que je disais sur la métamorphose. Si c'est réussi, les gens sortiront émus et troublés : émus parce qu'on réentend la force de ces témoignages et par la manière dont ils sont oralisés, et troublés parce que le regard porté sur cette jeunesse de SeineSaint-Denis va être ébranlé. La capacité de travail, le risque pris devant nous au plateau, la remise en cause de notre regard par leurs transformations physiques, tout cela crée du « et maintenant ». Et maintenant qu'est-ce qu'on fait de ces jeunes gens ? On n'y est pas du tout encore en France... La semaine dernière, lors d'une réunion avec des architectes pour la reconstruction de la Comédie de Saint-Étienne, je me suis surpris à remarquer que parmi eux il y en avait un prénommé Ali. Un. Dans les classes dirigeantes, la mixité sociale est loin d'être atteinte, à la différence du modèle anglo-saxon. D'une certaine manière, c'est de cela aussi que je parle dans le spectacle.

M. B. - À chaque coupe du monde de foot, on se félicite de la diversité de l'équipe de France dont on prend soudain conscience...

A. M. - Tout à fait, mais le foot, ce n'est pas du domaine intellectuel, alors que le théâtre représente une activité artistique, d'engagement, et je pense que cela va troubler. Je l'espère.

M. B. - Les lycéens "D'un 11 septembre à l'autre" ne sont finalement pas des "témoins ordinaires ». Du moins avec eux, tu questionnes plus que tu témoignes. Mais as-tu pensé à une possible instrumentalisation du spectacle au chœur des commémorations?

A. M. - Je ne sais pas. Il y aura de la curiosité, un petit phénomène, mais pour beaucoup de gens ça restera un atelier. Avec une suspicion de misérabilisme. Mais je crois en la démonstration par la preuve : la qualité de ce qui va se passer sur le plateau va surprendre, et si plusieurs personnes sont conquises et veulent se battre pour que ça continue, je serai content. Je trouve dommage que l'on ne fasse que six représentations. Le manque de volonté de nombreux partenaires m'a surpris et un peu blessé, je l'avoue.

M. B. - Vas-tu poursuivre ton travail de sensibilisation à Saint-Étienne, dans les quartiers et au sein même du théâtre-école de la Comédie?

A. M. - On fera le travail qu'on a fait en compagnie : aller vers ceux qui ne vont pas vers le théâtre. Au sein de l'école, j'aimerais mettre en place un dispositif similaire à celui de Sciences Po, une recherche volontaire, proche des «cordées de la réussite » qui existent déjà avec certains établissements scolaires. Ce projet serait mené avec l'école d'architecture. Comment faire pour qu'il y ait de la mixité et de la diversité dans les écoles d'art? C'est une recherche qui doit être volontaire. Pourquoi est-ce que des jeunes gens "issus de la diversité » selon la formule consacrée ne passent pas les concours? Cette année, sur cinq cents candidats au concours d'entrée de l'école, ils étaient moins de dix. Ils représentent peu de candidats, donc peu d'admis.

M. B. - Il n'existe pas encore de discrimination positive. 
A. M. - Je suis tout à fait partisan de cela, nous sommes en train de le construire. J'espère que certains participants au projet auront envie de continuer et de passer des concours d'écoles supérieures. Certains ont du talent et nous leur indiquerons les chemins à suivre pour continuer s'ils le veulent. Ça serait une fierté pour moi. Sans miroir aux alouettes : nous avons toujours été clairs sur le projet qui n'était pas de former des lycéens en comédiens professionnels. De même, il va falloir bien accompagner la fin de ce projet qui les a occupés pendant un an.

\section{Pour citer ce document}

Arnaud Meunier, " 43 lycéens de Seine-Saint-Denis : Manhattan sur le plateau », Agôn [En ligne], Dossiers, $\mathrm{HS} \mathrm{n}^{\circ} 1$ : Mettre en scène l'événement, D'un 11 septembre à l'autre, mis à jour le : 01/09/2011, URL : http://w7.ens-lsh.fr/agon/index.php?id=1796. 